\title{
A STUDY ON NUMERICAL MODELLING OF VECTOR-BORNE DISEASES
}

\author{
BUSHRA ABDULATEEF HASAN
}

(Received 18 January 2019; first published online 6 March 2019)

\begin{abstract}
2010 Mathematics subject classification: primary 92B05; secondary 34B60, 35Q92, 92D25, 92-08.
Keywords and phrases: numerical modelling, mosquito-borne diseases, malaria, Zika, dengue, deterministic modelling, diffusion, cross-diffusion, basic reproduction number, stability, bifurcation values, parameter identification, estimated parameters, sensitivity analysis.
\end{abstract}

This thesis is based on a study of three of the most serious mosquito-borne diseases, malaria, Zika and dengue, through numerical simulations. The research work has been conducted with a view to extending the understanding of the transmission of these vector-borne diseases which plays a significant role in their dynamics. An effort has been made to find strategies that can help to control these diseases.

The research work is based on deterministic modelling of vector-borne diseases. In this study, a series of numerical simulations are adopted to examine how the diffusion of human and mosquito populations affects the transmission of the malaria, Zika and dengue viruses.

First, a deterministic model, susceptible-infected-susceptible (SIS) for humans and susceptible-infected (SI) for mosquitoes, is considered in order to investigate the impact of self-diffusion on the spread of malaria with different modes of the transmission of the disease. This study is based on the numerical solutions of the differential equations under four different cases with different initial population distributions of both human and mosquitoes. Initial distributions of populations of humans and mosquitoes are observed to play a crucial role in the spread of the malaria disease. Subsequently, again with the SIS model, malaria with cross-diffusion and self-diffusion in the system is studied numerically. Five cases with the incorporation of cross-diffusion and self-diffusion and pure cross-diffusion are considered. Effects of cross-diffusion on the spread of malaria are shown to be quite significant.

Next, a susceptible-exposed-infected-recovered-susceptible-exposed-infected (SEIR-SEI) mathematical model of the Zika virus with diffusion is investigated

Thesis submitted to Swinburne University of Technology in May 2018; degree approved on 3 October 2018; supervisors Aaron Blicblau, Manmohan Singh, and David Richards.

(C) 2019 Australian Mathematical Publishing Association Inc. 
numerically. In the mathematical models, transmission of the virus, with and without sexual contact of humans, is also considered. Sexual contacts of the infected humans along with infected mosquitoes are found to be responsible for the spread of the Zika virus.

Finally, the vector-borne disease, dengue, is investigated. The parameters involved in the differential equation governing the model are re-estimated with the help of parameter identification and sensitivity analysis using the field data collected by Primary Industries of Victoria in the years 2003/2004. The parameters significant for the spread of the dengue virus have been analysed for their sensitivity based on the basic reproduction number, $R_{0}$. The SEIR dengue epidemic model, with newly estimated parameters and with the inclusion of diffusion in the system, is solved numerically.

BUSHRA ABDULATEEF HASAN, Department of Mathematics, Faculty of Science, Engineering and Technology,

Swinburne University of Technology, Hawthorn, Victoria 3122, Australia e-mail: bhasan@swin.edu.au 